\title{
Effects of advanced age upon astrocyte- specific responses to acute traumatic brain injury in mice
}

Alexandria N. Early ${ }^{1,2}$, Amy A. Gorman', Linda J. Van Eldik ${ }^{1,2,3}$, Adam D. Bachstetter ${ }^{1,2,3}$ and Josh M. Morganti ${ }^{1,2,3^{*}}$

\begin{abstract}
Background: Older-age individuals are at the highest risk for disability from a traumatic brain injury (TBI). Astrocytes are the most numerous glia in the brain, necessary for brain function, yet there is little known about unique responses of astrocytes in the aged-brain following TBI.

Methods: Our approach examined astrocytes in young adult, 4-month-old, versus aged, 18-month-old mice, at 1, 3, and 7 days post-TBI. We selected these time points to span the critical period in the transition from acute injury to presumably irreversible tissue damage and disability. Two approaches were used to define the astrocyte contribution to TBI by age interaction: (1) tissue histology and morphological phenotyping, and (2) transcriptomics on enriched astrocytes from the injured brain.
\end{abstract}

Results: Aging was found to have a profound effect on the TBl-induced loss of astrocyte function needed for maintaining water transport and edema-namely, aquaporin-4. The aged brain also demonstrated a progressive exacerbation of astrogliosis as a function of time after injury. Moreover, clasmatodendrosis, an underrecognized astrogliopathy, was found to be significantly increased in the aged brain, but not in the young brain. As a function of TBI, we observed a transitory refraction in the number of these astrocytes, which rebounded by 7 days postinjury in the aged brain. Transcriptomic data demonstrated disproportionate changes in genes attributed to reactive astrocytes, inflammatory response, complement pathway, and synaptic support in aged mice following TBI compared to young mice. Additionally, our data highlight that TBI did not evoke a clear alignment with the previously defined "A1/A2" dichotomy of reactive astrogliosis.

Conclusions: Overall, our findings point toward a progressive phenotype of aged astrocytes following TBI that we hypothesize to be maladaptive, shedding new insights into potentially modifiable astrocyte-specific mechanisms that may underlie increased fragility of the aged brain to trauma.

Keywords: Astrocytes, TBI, Aging, Clasmatodendrosis, Neuroinflammation

\section{Background}

Traumatic brain injury (TBI) is one of the most powerful environmental risk factors for the subsequent

\footnotetext{
* Correspondence: josh.morganti@uky.edu

'Sanders-Brown Center on Aging, University of Kentucky, Room 433, Sanders-Brown Bldg., 800 S. Limestone Street, Lexington, KY 40536, USA

${ }^{2}$ Department of Neuroscience, University of Kentucky, Lexington, KY 40536, USA

Full list of author information is available at the end of the article
}

development of Alzheimer's disease (AD) and related dementias [1-7]. Despite the aged population being at the greatest risk for acquiring a TBI, as well as the significant and long-lasting neurologic sequelae following the initial trauma in the aged population, current knowledge regarding how the aged brain responds to TBI remains disproportionately low, especially with respect to cellspecific responses. In the current study, we focused on

(c) The Author(s). 2020 Open Access This article is licensed under a Creative Commons Attribution 4.0 International License, which permits use, sharing, adaptation, distribution and reproduction in any medium or format, as long as you give appropriate credit to the original author(s) and the source, provide a link to the Creative Commons licence, and indicate if changes were made. The images or other third party material in this article are included in the article's Creative Commons licence, unless indicated otherwise in a credit line to the material. If material is not included in the article's Creative Commons licence and your intended use is not permitted by statutory regulation or exceeds the permitted use, you will need to obtain permission directly from the copyright holder. To view a copy of this licence, visit http://creativecommons.org/licenses/by/4.0/ The Creative Commons Public Domain Dedication waiver (http://creativecommons.org/publicdomain/zero/1.0/) applies to the data made available in this article, unless otherwise stated in a credit line to the data. 
the response of astrocytes to TBI in the aged brain to begin to elucidate several cell-specific dynamics. It is well known that astrocytes play a critical role in maintaining homeostasis in the CNS tightly regulating ATP, glucose and glutamate, and synaptic pruning and function, while also serving a crucial role for blood brain barrier (BBB) integrity and cerebral blood flow (CBF) [8-15]. However, as a response to injury or disease, astrocytes are able to rapidly respond, initially via ATP-mediated calcium signaling, in a generalized description referred to as reactive astrogliosis [16-18]. In the young adult brain, contemporary evidence has shown that astrocytes play a critical neuroprotective role following $\mathrm{TBI}$, as chemically ablating the vast majority of $\mathrm{GFAP}^{+}$astrocytes has been shown to exacerbate neuronal loss and perpetuate inflammatory response [19], owing to several intrinsic functions of astrocytes in mediating damage repair following TBI.

A continuing growth of supporting evidence implicates astrocytes as active participants in the multicellular networked responses potentially underlying, resolving, or exacerbating CNS diseases and injury [20,21]. As a variety of roles of astrocytes within the disease or injury milieu are being identified, it is becoming clearer that astrocyte response is rather heterogeneous [17, 20, 22-24], with implications pointing toward a diversity of graded responses [25]. Critically, among these responses are multiple domains encompassing inflammatory response, tissue protection, vascular response, as well as neuronal functionality [26]. However, despite the recognition of the diverse actions of astrocytes in response to trauma, relatively little information exists on how normal aging may alter the capacity of astrocytes to mount an appropriate response to TBI. Recent evidence has shown, as a consequence of normal aging, the brain acquires a chronically elevated inflammatory phenotype, which has been shown to negatively impact astrocyte function, leading toward their increased inflammatory bias [27-29]. Aged astrocytes were also shown to disproportionately respond to an inflammatory challenge (e.g., LPS), compared to young [28]. Collectively, these works proposed that the aged brain promotes a "neurotoxic" bias in astrocytes predominated by a pro-inflammatory response, with permissive phenotypes supporting increased synaptic clearance and neuronal damage [28, 29]. In parallel, we and others have recently demonstrated that the aged brain promotes exacerbated neuroimmune responses following TBI [30-32]. However, the understanding of the complex intrinsic and extrinsic mechanisms governing astrocyte-specific responses to TBI remains limited, especially in the aged brain. Ultimately, examining TBI in aged animal models is critically necessary to begin to unravel pathophysiological mechanisms likely to be found in the population at greatest risk for acquiring a TBI. Therefore, in this study we aimed to define astrocyte-specific morphological and transcriptional phenotypes using wellcharacterized homeostatic and disease-associated substrates.

\section{Methods \\ Animals}

All experiments were conducted in accordance with the National Institutes of Health Guide for the Care and Use of Laboratory Animals and were approved by the Institutional Animal Care and Use Committee of the University of Kentucky. Young adult (3-month-old; Jackson Laboratory) and aged adult (17-month-old; NIA aged rodent colony) male and female C57BL6 mice were used for all experiments. Prior to experimental procedures, all mice were acclimated to housing conditions at the University of Kentucky for approximately 4 weeks. All mice were group housed 4-5 per cage in individually ventilated cages, in environmentally controlled conditions with standard light cycle $\left(14: 10 \mathrm{~h}\right.$ light to dark cycle at $\left.21^{\circ} \mathrm{C}\right)$ and provided food and water ad libitum. Animals' ages at the time of surgery were approximately 4 or 18 months. Groupings for each endpoint and interval utilize an aggregated gender design; distributions of sex within each group/interval are detailed below.

\section{Surgical procedure}

All animals were randomly assigned and divided as equally as possible between sexes to their treatment group/endpoints. Animals were anesthetized with 2.5\% isoflurane before having their scalp shaved. Mice were maintained with $2.5 \%$ isoflurane via a non-rebreathing nose cone coupled to a passive exhaust system connected to a stereotaxic surgical frame (Stoelting). Animals' heads were secured to the stereotaxic frame using Delrin non-traumatic ear bars (Stoelting), eye ointment was applied, and scalps disinfected using betadine solution. Animal temperature during surgical procedures was maintained by a heating pad set to $37{ }^{\circ} \mathrm{C}$. A midline incision was made through the scalp to expose the skull. All mice, sham and TBI groups, received a craniectomy approximately $3.5 \mathrm{~mm}$ in diameter using a microburr electric drill with the center point to the coordinates of $-2.0 \mathrm{~mm}$ (anteroposterior), $2.0 \mathrm{~mm}$ (mediolateral), with respect to bregma, resultant bone flap was discarded for all mice. The controlled cortical impact (CCI) injury was reproduced using the Leica electromagnetic impactor and a $3.0-\mathrm{mm}$ convex tip, as we have previously described [30, 33, 34]. Impact parameters were as follows: impact velocity of $4.0 \mathrm{~m} / \mathrm{s}$, dwell time of $0.3 \mathrm{~s}$, to a depth of $-0.9 \mathrm{~mm}$, and rotated $20^{\circ}$ on the vertical axis to match the curvature of the brain, resulting in an impact force of $0.028 \mathrm{~N}$. These impact parameters align with previously suggested guidelines for $\mathrm{CCI}$ injury 
severity to be classified as mild to moderate in severity [35]. Following surgery, scalps were closed using surgical staples, and mice were transferred to a recovery cage placed on top of a heating pad. Animals were visually monitored until they were fully ambulatory, as exhibited by the resumption of movement and grooming behaviors. Surgical procedures were conducted in batches, such that mice were killed for all endpoints at approximately the same time during each day. Sham surgical controls were killed to match CCI post-surgical intervals; therefore, shams represent a mix of 1,3, and 7-day intervals. All animals fully recovered from surgical procedures.

\section{Histological tissue collection and preparation}

At the predefined post-injury interval, both young $(n=4$ group/interval; $2: 2 \quad$ $:$ : + ) and aged ( $n=5$ group/interval; 3:2 0 :우) mice were anesthetized with $5.0 \%$ isoflurane before exsanguination and transcardial perfusion with ice-cold phosphate buffered saline (PBS), followed by $4 \%$ paraformaldehyde in PBS. Immediately following perfusion, whole brain tissues were removed and bisected along the midline, and the ipsilateral hemisphere was drop-fixed in $4 \%$ buffered paraformaldehyde (PFA) overnight at $4{ }^{\circ} \mathrm{C}$. Following the overnight post fixation, brain hemispheres were transferred into $30 \%$ sucrose for at least 4 days before coronally sectioned on a microtome to a $30 \mu \mathrm{m}$ thickness. Tissue sections were serially collected at a 1:10 interval into $2 \mathrm{~mL}$ screw cap tubes containing $30 \%$ sucrose and stored at $-20{ }^{\circ} \mathrm{C}$.

\section{Astrocyte immunofluorescent labeling}

One $2 \mathrm{~mL}$ tube comprising every tenth serial section through the ipsilateral hemisphere was stained per animal using the following procedures. Sections were washed at room temperature three times for $10 \mathrm{~min}$ each in PBS, followed by $1 \mathrm{~h}$ of blocking using $10 \%$ normal goat serum (NGS) in PBS containing 0.1\% TritonX-100 (PBST). Subsequently, sections were incubated with primary antibodies against GFAP (Thermo Fisher Scientific Cat\# 13-0300, RRID:AB_2532994, 1:800 dilution), S100 $\beta$ (Agilent Cat\# GA50461-2, RRID:AB_2811056, 1:400 dilution), Vimentin (Millipore Cat\# AB5733, RRID:AB 11212377, 1:400 dilution), or aquaporin-4 (Aqp4) (Sigma-Aldrich Cat\# HPA014784, RRID:AB_1844967, 1: 400 dilution) diluted in $1 \%$ NGS-PBST. Primary incubations were conducted overnight, approximately $16 \mathrm{~h}$, at 4 ${ }^{\circ} \mathrm{C}$. Sections were subsequently washed at room temperature 5 times in PBST for 5 min each. Using appropriate detection antibodies, diluted in 1\% NGS-PBST, for rabbit (Thermo Fisher Scientific Cat\# A-11034, RRID:AB_2576217), rat (Thermo Fisher Scientific Cat\# A-21094, RRID:AB_2535749), or chicken (Thermo Fisher Scientific Cat\# A-11041, RRID:AB_2534098) each at 1:200 dilutions, sections were incubated for $2 \mathrm{~h}$ at room temperature. Tissue sections were subsequently washed 3 times in PBST for $5 \mathrm{~min}$ each, followed by 2 washes in PBS for 5 min each. Sections were mounted on Superfrost slides (Fisher \#12-550-15) and allowed to air dry in the dark overnight before coverslipping with Antifade mounting medium with DAPI (Vector \#H1200). Slides were sealed with nail polish and allowed to dry overnight.

\section{Cellular imaging and quantification}

Immunofluorescently labeled sections were imaged using a Zeiss Axio Scan Z1 digital slide scanner at $\times 20$ magnification. Digital images of each slide and its sections containing dorsal hippocampus ( $n=6-8$ per animal) were analyzed for threshold-defined pixel-positive area fraction using the Halo Image analysis software (Indica Labs, v2.3.2089.34) utilizing the AreaQuantification v1.0 algorithm. For all analyses, an investigator blinded to the study conditions outlined anatomical regions consisting of the dorsal hippocampus and neocortex. The positive staining baseline was thresholded against representative young-sham positive staining, such that any pixel at that intensity or greater (e.g., brighter) was quantified. The number of positive pixels was normalized per area outlined for each section to account for outlined region-toregion area variability. All sections were batch analyzed using the stored parameters in the algorithm. For quantification of clasmatodendrosis, dorsal hippocampal structures were outlined as above; however, a blinded investigator used Halo Annotation tool to manual mark each cell. Marking of clasmatodendrosis was first defined by identification of single $\mathrm{DAPI}^{+} \mathrm{GFAP}^{+}$dual-positive astrocytes. Single astrocytes were then examined for $\mathrm{GFAP}^{+}$filaments that exhibited S100 $\beta^{+}$swellings, which colocalized along that cell's filaments. For each identified cell, the blinded investigator annotated each cell on its $\mathrm{DAPI}^{+}$nucleus in this manner. The number of annotations was normalized per area for each tissue section. For all HALO analyses, there were between 6-8 sections per animal outlined and quantified for their respective histological markers. Additionally, representative confocal images of clasmatodendrosis in astrocytes were acquired using a Nikon C2Plus Confocal Microscope. Representative renderings of the GFAP, Vimentin, and S100 $\beta$ positive surfaces were created using Imaris (v9.3.1, Oxford Instruments).

\section{Astrocyte enrichment and RNA isolation}

At the prescribed interval, mice $(n=4$ group/interval, per age; $2: 2 \quad \delta$ : 9 ) were anesthetized with $5.0 \%$ isoflurane before exsanguination and transcardial perfusion with ice-cold Dulbecco phosphate buffered saline (DPBS; Gibco \# 14040133). Following perfusion, brain tissues 
were removed and quickly dissected to isolate the ipsilateral pericontusion neocortex, approximately $3 \mathrm{~mm}$ in diameter (or analogous region in sham mice) and dorsal hippocampal structure. Dissected tissues were immediately transferred into gentleMACS C-tube (Miltenyi \#130-093-237) containing Adult Brain Dissociation Kit (ADBK) enzymatic digest reagents (Miltenyi \#130-107677) prepared according to manufacturer's protocol. Tissues were dissociated using the "37C_ABDK" protocol on the gentleMACS Octo Dissociator instrument (Miltenyi \#130-095-937) with heaters attached. After tissue digestion, cell suspensions were processed for debris removal and filtered through $70 \beta \mu \mathrm{m}$ mesh cell filters following manufacturer's suggested ABDK protocol. The resultant cell suspension was used for magnetic bead enrichment for astrocytes (Miltenyi \#130-097-678) following manufacturer's suggested procedures, however utilizing three MS-columns (Miltenyi \#130-042-201) to enhance purification. Washes were conducted using AstroMacs separation buffer (Miltenyi \#130-117-336). The astrocyte cell surface antigen 2 positive fraction (ACSA-2 ${ }^{\text {pos }}$ ) was collected into a separate tube, then centrifuged for $5 \mathrm{~min}$ at $1000 \times g$ at $4{ }^{\circ} \mathrm{C}$ to pellet cells. Both the flow ACSA- $2^{\text {neg }}$ and astrocyte enriched ACSA$2^{\text {pos }}$ fraction were collected from a subset of samples to validate putative gene enrichment efficiency.

Following centrifugation, supernatant was carefully aspirated, and cell pellets were lysed using RLT+ buffer containing $1 \% \beta$-mercaptoethanol using the Qiagen RNeasy + Micro Kit (Qiagen \#74034), following manufacturer's suggested protocol for RNA isolation. RNA quantity and quality (e.g., 260:280 ratio) was assessed using NanoDrop 2000 spectrophotometer, and approximately $25 \mathrm{ng}$ of total RNA was converted to cDNA using High-Capacity cDNA Reverse Transcription Kit (Applied Biosystems \# 4368813). Resultant cDNA was stored at $80{ }^{\circ} \mathrm{C}$ until assayed.

\section{Gene expression arrays}

Multiplexed gene expression profiling was conducted on a ViiA7 qRT-PCR machine (Applied Biosystems) using a custom built Taqman low density array card (Applied Biosystems) consisting of 44 genes of interest plus one housekeeping gene (HPRT, Mm00446968_m1). Genes on the array were curated from recent publications that previously determined putative astrocyte-specific responses to injury, disease, and aging [28, 29, 36]. Taqman gene probes were as follows: Amigo2 (Mm00662105_s1), Apoe (Mm01307193_g1), B3gnt5 (Mm01952370_u1), C1qa (Mm00432142_m1), C1qb (Mm01179619_m1), C3 (Mm01232779_m1), CD109 (Mm00462151_m1), CD44 (Mm01277161_m1), Clcf1 (Mm01236492_m1), Clu (Mm01197002_m1), CXCL10 (Mm00445235_m1), Emp1 (Mm00515678_m1), Fbln5
(Mm00488601_m1), Fkbp5 (Mm00487406_m1), Gbp2 (Mm00494576_g1), GFAP (Mm01253033_m1), Ggta1 (Mm01333302_m1), Gpc4 (Mm00515035_m1), Gpc5 (Mm00615599_m1), H2-T23 (Mm00439246_g1), Iigp1 (Mm00649928_s1), Lcn2 (Mm01324470_m1), Maoa (Mm00558004_m1), Osmr (Mm01307326_m1), Psmb8 (Mm00440207_m1), Ptgs2 (Mm00478374_m1), Ptx3 (Mm00477268_m1), S100b (Mm00485897_m1), S1pr3 (Mm02620181_s1), Serpina3n (Mm00776439_m1), Serping1 (Mm00437835_m1), Sparcl1 (Mm00447784_m1), Sphk1 (Mm00448841_g1), Stat3 (Mm01219775_m1), Steap4 (Mm00475405_m1), Tgm1 (Mm00498375_m1), Thbs1 (Mm00449032_g1), Thbs2 (Mm01279240_m1), Thbs4 (Mm03003598_s1), Timp1 (Mm01341361_m1), Tm4sf1 (Mm00447009_m1), and Vim (Mm01333430_ m1). cDNA from each sample was diluted with TaqMan Gene Expression Master Mix (Applied Biosystems \# 4369016) according to manufacturer's protocol. Relative gene expression ratios were analyzed using the $2^{-\Delta \Delta C T}$ method, with young-sham as the reference group. All gene expression ratios were $\log _{2}$ transformed.

\section{Individual TaqMan assays}

Single assay reactions utilized for ACSA- $2^{\text {pos }}$ sample enrichment calculations were also conducted on the ViiA7 using cDNA diluted with TaqMan Fast Advanced MasterMix (Applied Biosystems \#4444557); HPRT (Mm00446968_m1), Aldh1l1 (Mm03048957_m1), Tmem119 (Mm00525305_ m1), Dlg4 (Mm00492193_m1), Klk6 (Mm00478322_m1), and Nostrin (Mm00724960_m1). Relative gene expression ratios were analyzed using the $2^{-\triangle \Delta C T}$ method, with the ACSA- $2^{\text {neg }}$ fraction as the reference group. All gene expression ratios were $\log _{2}$ transformed.

\section{Statistical analyses}

All data were captured in a blinded manner, with the investigator unaware of groupings. The data codes were revealed, and groupings assigned only after all data for each endpoint were captured. Statistical analyses were conducted in JMP Pro (v14.0), along with figures created in GraphPad Prism (v8.0). Pre-planned contrasts to examine the effect of age by post-surgical interval (histological and gene expression) were conducted using ANOVA with Sidak's multiple comparison post hoc analysis. For heatmap of gene expression data, column (e.g., gene) data were standardized via $z$-score transformation; data were examined as a function of deviation from young sham values using ANOVA with Dunnet's multiple comparison post hoc analyses. For representative analyses examining the effect of age within a post-injury interval, ANOVA with Sidak's multiple comparison post hoc analysis was used. Multidimensional reduction was conducted using principal components analysis (PCA) with Varimax orthogonal rotation for gene expression 
data. PCs were considered of interest using cutoffs of $>$ 1 for eigenvalue and Scree plot criteria. Resultant PC scores were calculated using a regression method, and their PC loadings were represented using arrows, where gauge (e.g., thickness) is indicative of loading magnitude, coloring heat of orange to blue indicates loading value with orange indicating greater magnitude, and blue representing more minimal magnitudes. Loading magnitudes with a cutoff of $>|0.45|$ were graphically represented for interpretation. Significance for all measures was assessed at $p<0.05$.

\section{Results}

TBI elicits delayed reactive gliosis that is protracted in the aged brain

To examine whether reactive astrogliosis is sensitive to age at the time of injury, as well as the progression of time after injury, we examined GFAP reactivity in the ipsilateral dorsal hippocampal formation and adjacent neocortex. GFAP, an intermediate filament protein along with Vimentin, are classical histological markers for assessing reactive astrogliosis, which are consistently assessed to gauge trauma-associated responses of astrocytes [37-39]. Further, utilizing these markers is sufficient to assess the degree of homeostatic disturbance, as GFAP and Vimentin have shown a somewhat linear relationship with severity of and proximity to injury in the brain [25]. We found a TBI-induced increase in both hippocampal and cortical $\mathrm{GFAP}^{+}$astrogliosis over the first 7 days post-injury in both young and aged animals (Fig. 1). In the hippocampus, young mice showed the highest magnitude response by 3 days post-injury and a return toward baseline thereafter, whereas the aged cohort exhibit a protracted response with the highest magnitude response at 7 days post-injury (Fig. 1a, b). Similar temporal responses were also observed in the neocortex for both ages; however, young mice maintained reactivity through 7 days post-injury, which was exacerbated in the aged condition (Fig. 1a, c). Similarly, we quantified Vimentin in the context of another reactive marker associated with astrocytes: $\mathrm{S} 100 \beta$. Although $\mathrm{S} 100 \beta$ is predominantly expressed by astrocytes in the CNS, it is not exclusive to this cell type [40]; therefore, examining the co-labeled fraction with Vimentin allowed us to assess its astrocyte-specific reactivity. Examining the colocalized area (e.g., dual positive for Vimentin and S100ß) in the same hippocampal and neocortical regions as above, our data demonstrated that in the young brain, these dual-positive astrocytes are less reactive to TBI (Fig. 2a, b), compared to the aged brain at all post-injury intervals for the hippocampus. Comparatively, the neocortex demonstrated a delayed reactive profile, such that appreciable dual-labeled astrocytes were not present until the
3- to 7-day intervals, which interestingly did not show an age-related bias (Fig. 2c).

\section{Advanced age promotes accumulation of clasmatodendrosis in astrocytes in the HPC, which was not overtly affected by TBI}

First reported by Alois Alzheimer and later coined by Cajal [41], clasmatodendrosis is typified by the beading and diminishment of astrocyte projections, paired with vacuolization and swelling of the cytoplasm [42-44]. This astrocyte pathology has been observed in AD, Binswanger's mixed dementia, aging, and ischemic brain tissues [43-47]. Furthermore, recent evidence in postmortem analyses of chronic traumatic encephalopathy (CTE) has demonstrated trauma-induced accumulation of a morphologically distinct subset of reactive astrocytes [48]. Characteristically, these astrocytes had a beaded "pearl on a string" degenerative morphology. We observed a significant accumulation of these degenerative-like astrocyte morphologies in the molecular layer of the stratum radiatum of the hippocampus (Fig. 3a) in our injured aged mice, and these astrocytes were found in aggregates (Fig. 3b). Our triple labeling method revealed $S 100 \beta^{+}$Vimentin ${ }^{+}$dualpositive vacuolization along $\mathrm{GFAP}^{+}$filament tracks and somatic hypertrophy (Fig. 3c) consistent with morphological hallmarks attributed to clasmatodendrosis of astrocytes. Quantification of the numbers of astrocytes exhibiting clasmatodendrosis demonstrated that only aged mice showed these morphologically distinct astrocytes in the HPC, and that TBI in the aged mice induced a decrease in these astrocytes over the 3-day post-injury period, which returned to pre-TBI levels by 7 days post-injury (Fig. 3d).

\section{Astrocyte endfoot integrity is diminished by TBI and advanced age}

Consistent evidence has implicated astrocytes as a critical component of the inflammatory response to CNS trauma [49], mediated in part by their polarization of endfeet around vascular surfaces throughout the brain. Classically, the polarity of astrocytes to perivascular localization has been assessed by analysis of aquaporin-4 (Aqp4), which is a water channel expressed exclusively in the CNS by astrocytes [50] and is a critical component of waste clearance pathways. Given these critical physiological properties, we quantified the total area of Aqp4 staining in the ipsilateral hippocampus and neocortex of our young and aged cohorts. Our data demonstrate that there is a trend for aged mice (sham) to have decreased Aqp4 positive area in the hippocampus, relative to young shams (Fig. 4a). However, after TBI, we observed a significant change in Aqp4 only in the aged mice and only at 3 days post-injury (Fig. $4 \mathrm{~b}$ ). The TBIinduced rarefaction of $\mathrm{Aqp}_{4}^{+}$area seen at 3 days post- 


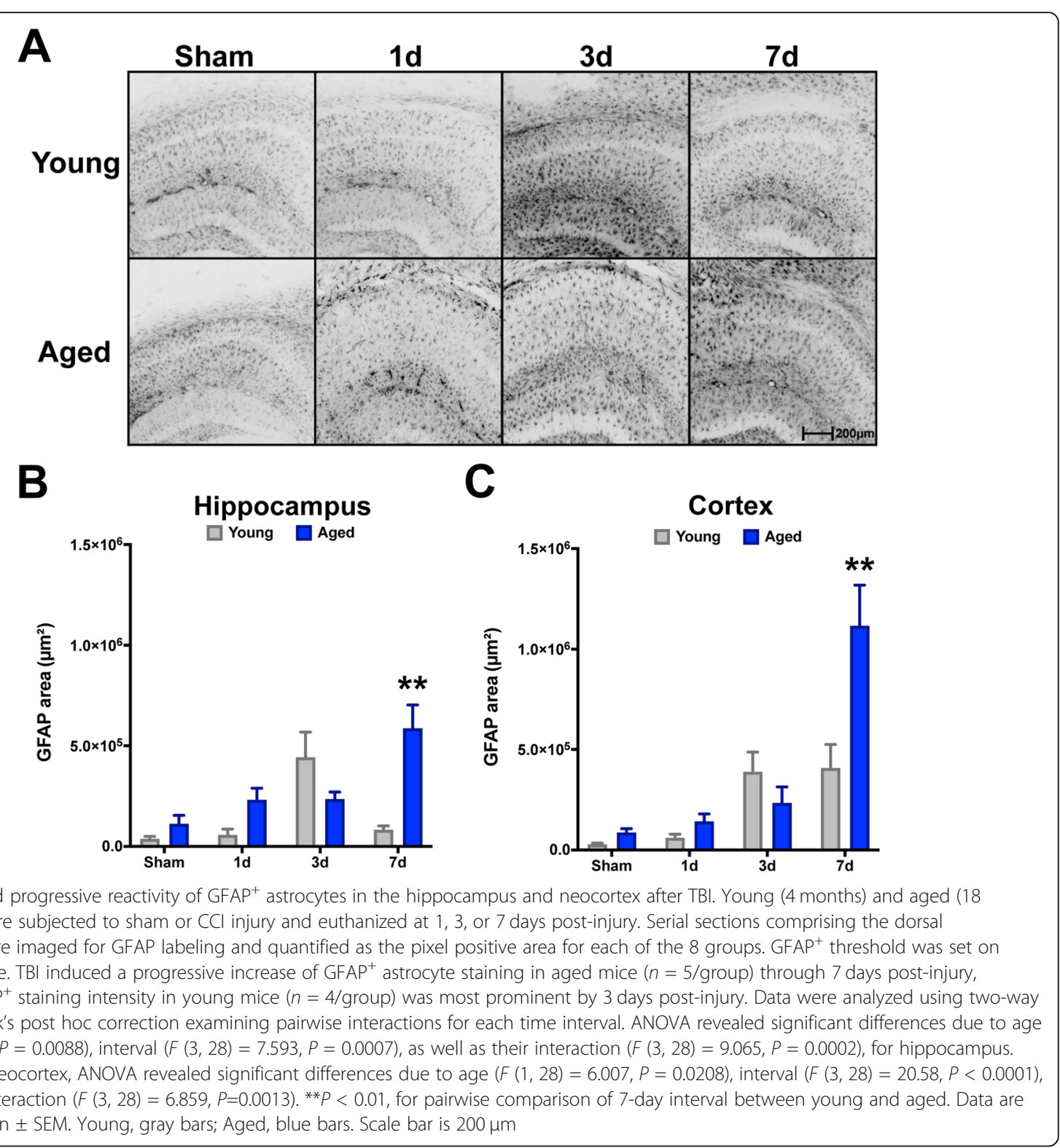

injury returned to similar levels of their young comparators by 7 days post-injury. Comparatively, the neocortical response demonstrated a trend for increased Aqp $4^{+}$area at 1 day post-injury for both age groups, with a similar reduction found in the aged cohort by day 3 (Fig. 4c).

\section{Mirroring responses profiling TBI-induced astrocyte transcriptional responses across representative A1/A2 categorizations}

Recent work has defined astrocyte-specific responses to either generic, microglia/lipopolysaccharide (LPS) mediated, or middle cerebral artery occlusion (MCAO) stimuli in neonate rodents to fall within categorical bins of "Pan-reactive," "A1," $\beta$ or "A2," respectively $[36,51]$. Given that TBI elicits upregulation and reactivity of a variety of convergent pathways attributed to both microglial and ischemic responses, we examined a subset of these transcriptional markers that were prominent in defining these categorizations, with an additional focus on genes associated with regulating synaptic function [29]. Therefore, we wanted to determine whether TBI and/or age affected transcriptional responses of astrocytes. In order to examine putative astrocyte responses in the context of age, injury, and interval, we utilized a magnetic bead enrichment protocol (Fig. 5a) previously demonstrated by others to enrich astrocytes from adult CNS tissues [52-54]. Using this procedure, we validated the enrichment efficiency by examining genes enriched for putative CNS subsets of astrocytes (Aldh1l1), neurons (Dlg4), oligodendroglia (Klk6), microglia (Tmem119), and endothelia (Nostrin). The results (Fig. 5b) 

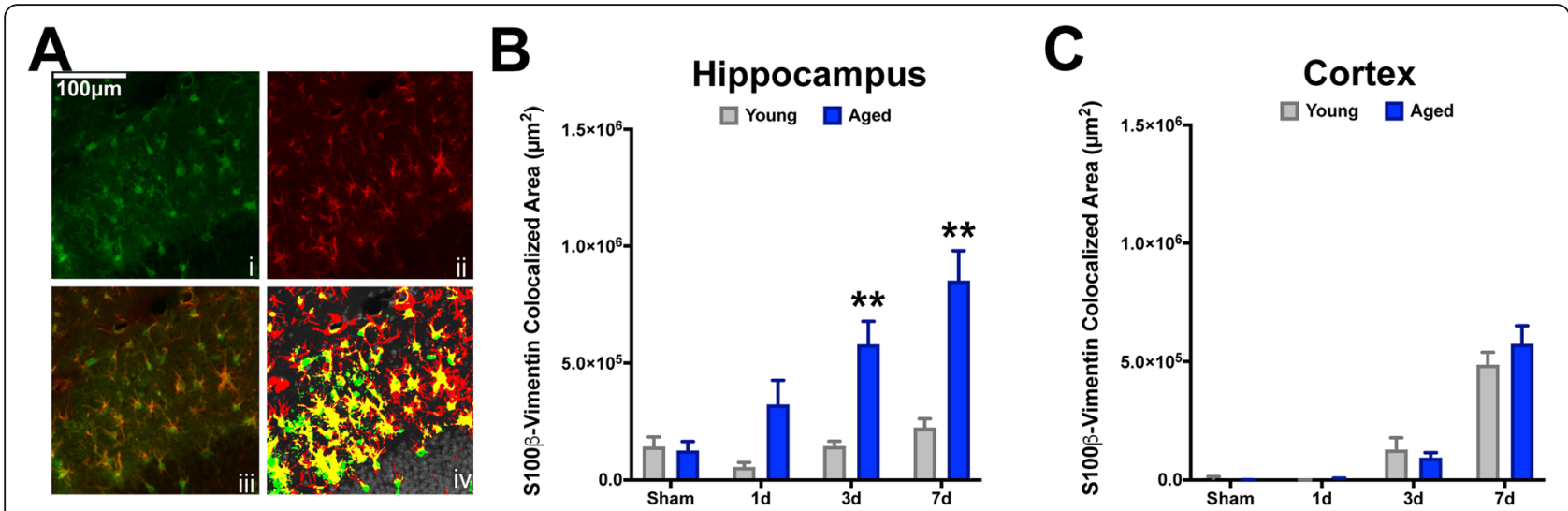

Fig. 2 Aged mice display exacerbated $S 100 \beta^{+} V_{i m}{ }^{+}$reactive astrogliosis in the hippocampus but not the neocortex following TBI. Serial sections comprising the dorsal hippocampus were imaged for $S 100 \beta$ (i) and Vimentin (ii) as a colocalization image (iii). Pixel positive area was defined for each stain using young sham's levels. HALO colocalization algorithm was used to compute the total colocalized area for both S100 (green, iv) and Vimentin (red, iv), which was represented as the dual-positive staining fraction (yellow, iv). Dual-positive astrocytes were increasingly reactive as a function of time after injury for aged mice, which show a progressive accumulation peaking at 7 days post-injury. Comparatively, young mice display relatively little change as a result of TBI at any time post-injury. ANOVA revealed significant differences in the hippocampus due to age $(F$ $(1,28)=33.61, P<0.0001)$, interval $(F(3,28)=10.38, P<0.0001)$, and their interaction $(F(3,28)=5.831, P=0.0032)$. However, no significant effects for age, interval, or their interaction were observed for these measures in the neocortex. ${ }^{* *} P<0.01$ for pairwise comparisons of 3-day and 7-day intervals between young and aged mice. $n=4-5 /$ group. Data are presented as mean \pm SEM. Young, gray bars; Aged, blue bars. Scale bar is $50 \mu \mathrm{m}$

demonstrated marked enrichment of Aldh1l1 in the ACSA $-2^{+}$fraction relative to the markers for other cell types.

Using RNA from magnetic bead enriched astrocyte fractions, we profiled 44 genes that spanned 4 categorical bins attributed to astrocyte biological and pathological profiles (Fig. 6a). Overall, our findings indicate that neither TBI nor advanced age were particularly constrained to either "A1" or "A2" categorical bins, with significant gene expression changes observed predominantly in the "Pan-reactive" and "Synaptic modifying" motifs (Fig. 6a, black and magenta headers). However, we did observe the induction of several genes in the "MCAO-associated" bin (Fig. 6a, green header). Notably, there are minimal transcriptional changes attributed to TBI or advanced age in the reactive microglia/LPS-/“A1"-associated phenotype (Fig. 6a, blue header). Although neither TBI nor age was found to predominantly align with these categorizations, we did observe several interesting dynamics when examining the effect of age at each temporal post-surgical interval. For CD44, CXCL10, C1qa, Amigo2, Ligp1, and B3gnt5, we observed significant differences occurring at 1 day post-injury for aged astrocytes, compared to young (Fig. 6b). Notably, there appeared to be a plateau effect with these markers initiated at 1 day post-injury that had a protracted response in magnitude trends carrying through 7 days post-injury (Fig. 6b). Comparatively, expression of both GPC6 and Cd109 was consistently depressed in aged mice relative to their young counterparts at all time intervals and showed relatively little fluctuation as a function of post-injury interval (Fig. 6b).

\section{Examining astrocyte-specific transcriptional dynamics in} an unbiased multivariate approach

We have previously examined the brain's response to TBI focusing upon myeloid (e.g., microglia and monocytes) specific responses utilizing multivariate methods to delineate the effect of injury and time [33]. Herein, we have employed similar methods using principal components analyses (PCA) in an unbiased approach to examine how age, interval, and injury converge to drive the multidimensional responses of astrocytes in the injured milieu. This analysis yielded three orthogonal PCs, in total, accounting for $64.3 \%$ of the total variance in astrocyte transcriptional responses within the 46 genes examined (Fig. 7a). The PC1 module reflects gene expression shifts due to TBI as 1-, 3-, and 7-day groups that were largely distinct from their sham comparators. Interestingly, there is an initial divergence in this profile as a function of age (Fig. 7b) at the 1-day interval; however, by 7 days PC loadings between age groups were strikingly similar. The PC1 module was largely driven by CD44, Serpina3n, and Tgm1 gene expression profiles (Fig. 7c, PC1 loadings > 0.8). Comparatively, the PC2 module reflects the temporal dynamics of the post-injury intervals, where both young and aged astrocytes display similar temporal u-shaped trajectories, with an initial repressed response, which overshoots its baseline for young, but fails to return for aged (Fig. 7b). PC2 responses were largely driven by expression changes associated with Apoe, Maoa, Clu, S100b, Gpc5, and Sparcl1 (Fig. 7c, PC2 loadings > 0.8). Lastly, the PC3 module encapsulates gene expression profiles predominated by advanced age, as the sham, 1 day, 3 days, and 7 days aged 

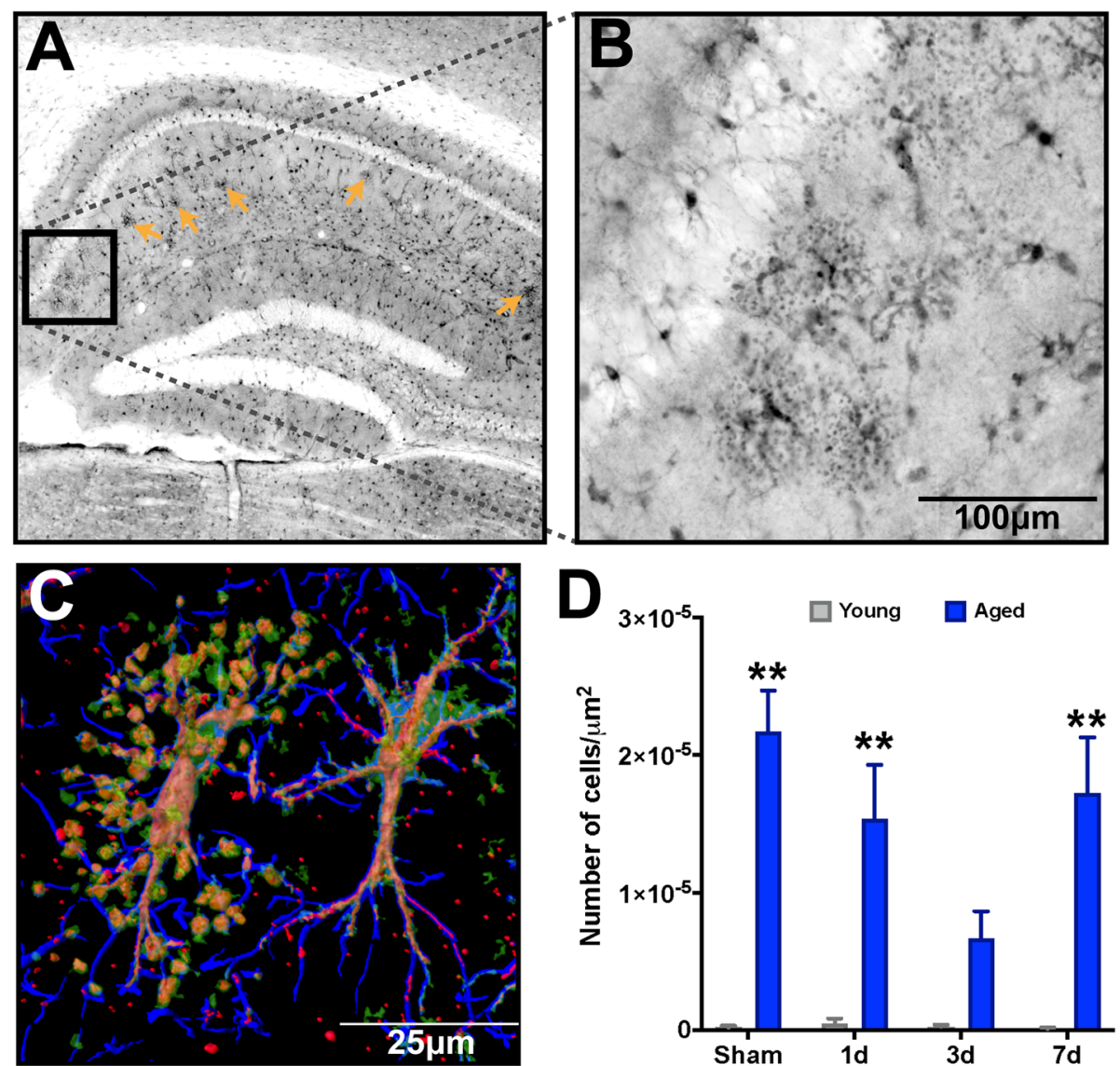

Fig. 3 Advanced age results in accumulation of clasmatodendrosis in the stratum radiatum of the dorsal hippocampus. S100 $\beta$ labeling reveals a significant accumulation of degenerative astrocytes. a Low power magnification of dorsal hippocampus shows the distribution of clasmatodendrosis localized within the stratum radiatum of the CA1 region of the hippocampus (orange arrows). b High power magnification shows the distinctive enlarged soma and vacuolization of processes distinctive to clasmatodendrosis for a representative cluster of astrocytes. $\mathbf{c}$ Imaris surface render of a confocal z-stack of GFAP (blue), S100ß (green), and Vimentin (Red) demonstrates an astrocyte with clasmatodendrosis (left) showing the co-localization of $\mathrm{S} 100 \beta^{+} V_{i m e n t i n}{ }^{+}$beads along GFAP ${ }^{+}$processes, and a reactive astrocye with non-degenerative morphology adjacent to it (right). $\mathbf{d}$ There is a significant accumulation of clasmatodendrosis in the aged brain, which did show some temporal response to $\mathrm{TBI}$ at 3 days post-injury; however by 7 days, levels return to approximate those in the uninjured condition. $n=4-5 /$ group. Data were analyzed using two-way ANOVA with Sidak-Holm post hoc correction examining pairwise interactions for each time interval. ANOVA revealed a significant main effect due to age $(F(1,28)=64.41, P<0.0001)$; however, neither interval nor their interaction were significantly different. ${ }^{*} P<0.01$ for pairwise comparisons of sham, 1-day, and 7-day interval between young and aged mice. $n=4-5 /$ group. Data are presented as mean \pm SEM. Young, gray bars; Aged, blue bars

astrocytes were starkly delineated in the multivariate plot (Fig. 7a, b), showing no overlap at any point, comparatively. Aged responses driving the PC3 module were largely attributed to CD109 (Fig. 7c, PC3 loadings > 0.8). Lastly, we examined the similarity between our dataset and a previously published RNAseq dataset by Anderson et al. that examined astrocyte-specific responses following spinal cord injury (SCI). We chose to examine the 3-day post-injury interval of our gene expression responses, because this time point showed several convergent responses across the endpoints described above. Using the mean log transformed fold change $(\log \mathrm{FC})$ from "SCI WT astrocytes" vs. "Uninjured WT astrocytes" from the Anderson et al. dataset, we plotted the mean $\operatorname{logFC}$ of our genes that had a PC loading $>|.45|$ from our 3
PCs for both young and aged astrocytes at the 3-day postinjury interval (Fig. 7d). Comparatively, our data follows remarkably similar expression profiles, particularly for PC1. Preservation of these trends between our current findings and those in the Anderson et al. dataset may potentially suggest a conservation of trauma-induced transcriptional profiles in astrocytes irrespective of CNS locale.

\section{Discussion}

Our study demonstrates that TBI induces a progressive increase of reactive astrogliosis in the aged hippocampus and neocortex. We demonstrated novel heterogeneity between reactive astrocytes' morphologies and agerelated clasmatodendrosis of astrocytes. Further, after 


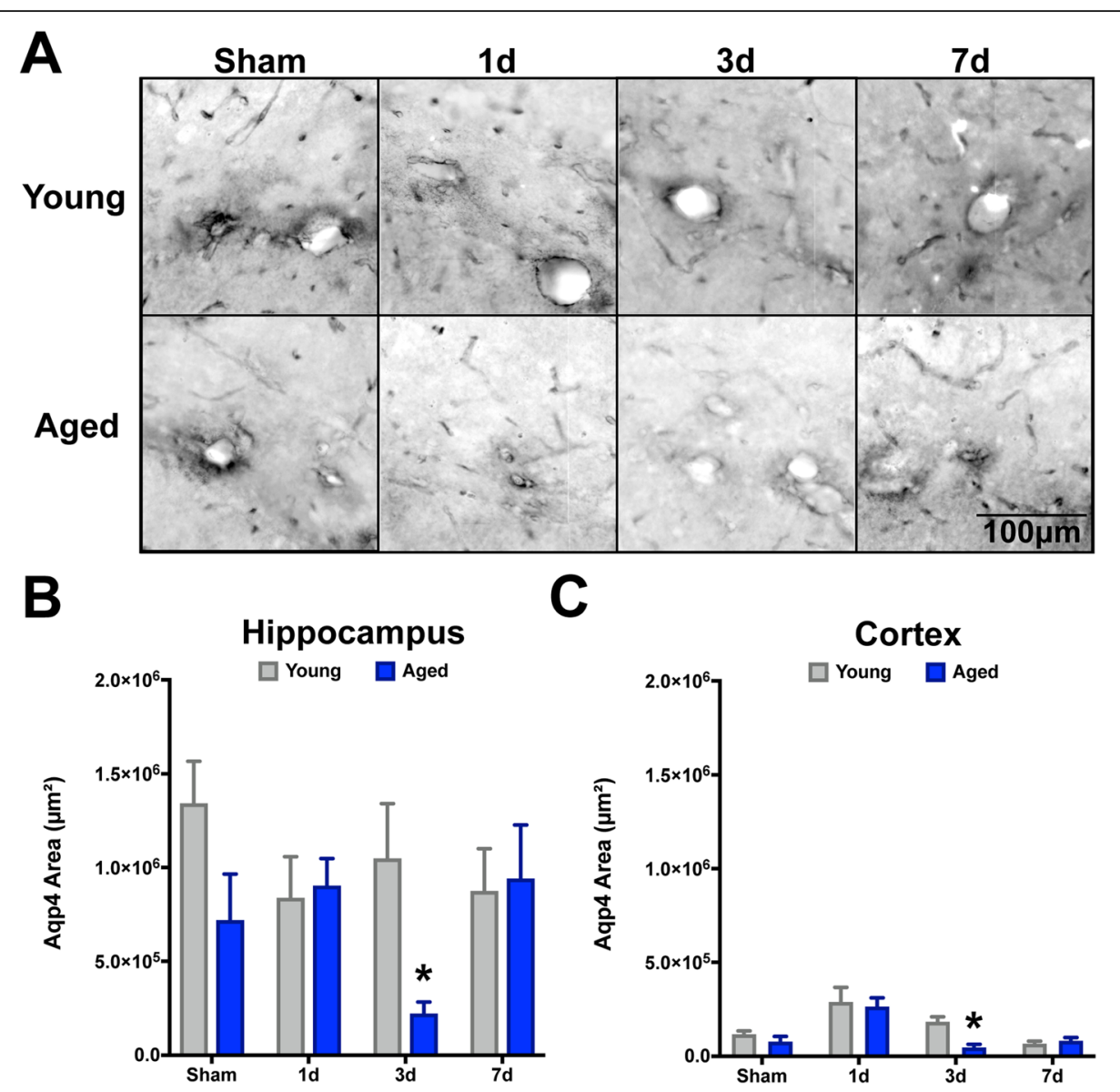

Fig. 4 Aquaporin-4 displayed a delayed transient response to TBI in the aged brain. Serial sections comprising the dorsal hippocampus were imaged for aquaporin-4 (Aqp4) labeling and quantified as the pixel positive area for each of the 8 groups. Aqp4 $4^{+}$threshold was set on young sham tissue. Overall, in the hippocampus there was a significant decrease in Apq4 staining due to advanced age $(F(1,28)=4.506, P=0.0435)$, which was significantly reduced compared to young at the 3 -day post-injury interval ( $\left.{ }^{*} p<0.05\right)$. This age-related loss of Aqp4 ${ }^{+}$recovered to sham-like levels at the 7-day post-injury interval. Additionally, for the hippocampus, there were no significant overall differences observed due to interval, nor an interaction effect. Comparatively, for the neocortex, there was not an age-related effect, but there was a significant effect due to interval $(F(3,28)=14.03, P<0.0001)$, which was demonstrated by significant alterations in aged mice at the 3-day interval, as well $(P=0.0473)$. Data were analyzed using two-way ANOVA with Sidak-Holm post hoc correction examining pairwise interactions for each interval. $n=4-5 / g r o u p$. Data are presented as mean \pm SEM. Young, gray bars; Aged, blue bars

dissecting the molecular phenotypes using a focused array, our current data demonstrate that TBI was not sufficient to align itself to previously defined molecular profiles associated with the "A1/A2" phenotypic bins $[28,36]$. However, using these unique gene expression markers did identify several novel responses of aged astrocytes to TBI. Unbiased multivariate analyses demonstrated that injury, interval, and age correlated with distinct gene expression profiles collectively pointing toward increased inflammatory response, decreased synaptic support, and increased pruning phenotypes of aged astrocytes following TBI, compared to young. These findings may suggest that astrocytes in the aged brain have lost key restraining-or acquired dysfunctional mechanisms, ultimately driving what we hypothesize to be a maladaptive response to TBI, compared to young.
In terms of the age-related progressive reactive astrocyte response, our findings parallel a previous report that examined GFAP reactivity in 21- to 24-month-old mice that received a CCI centered over the caudate putamen [55]. Further, although our data demonstrate an exacerbation of the $S 100 \beta$ response in the aged brain, the exact consequence of overactive $S 100 \beta$ in the aged brain remains unknown, as it has been implicated in a variety of roles in both a healthy and diseased CNS [56]. Whether aged astrocytes are propagating damage-associated molecular patterns (DAMPs) via S100 $\beta$ [57] or this signaling is potentiating previously defined neurosupportive roles [58-61] remains to be determined. Recent work has demonstrated distinct molecular profiles in astrocytes between anatomical regions [28, 29], suggesting regionally restricted functional roles. To this end, we also observed 


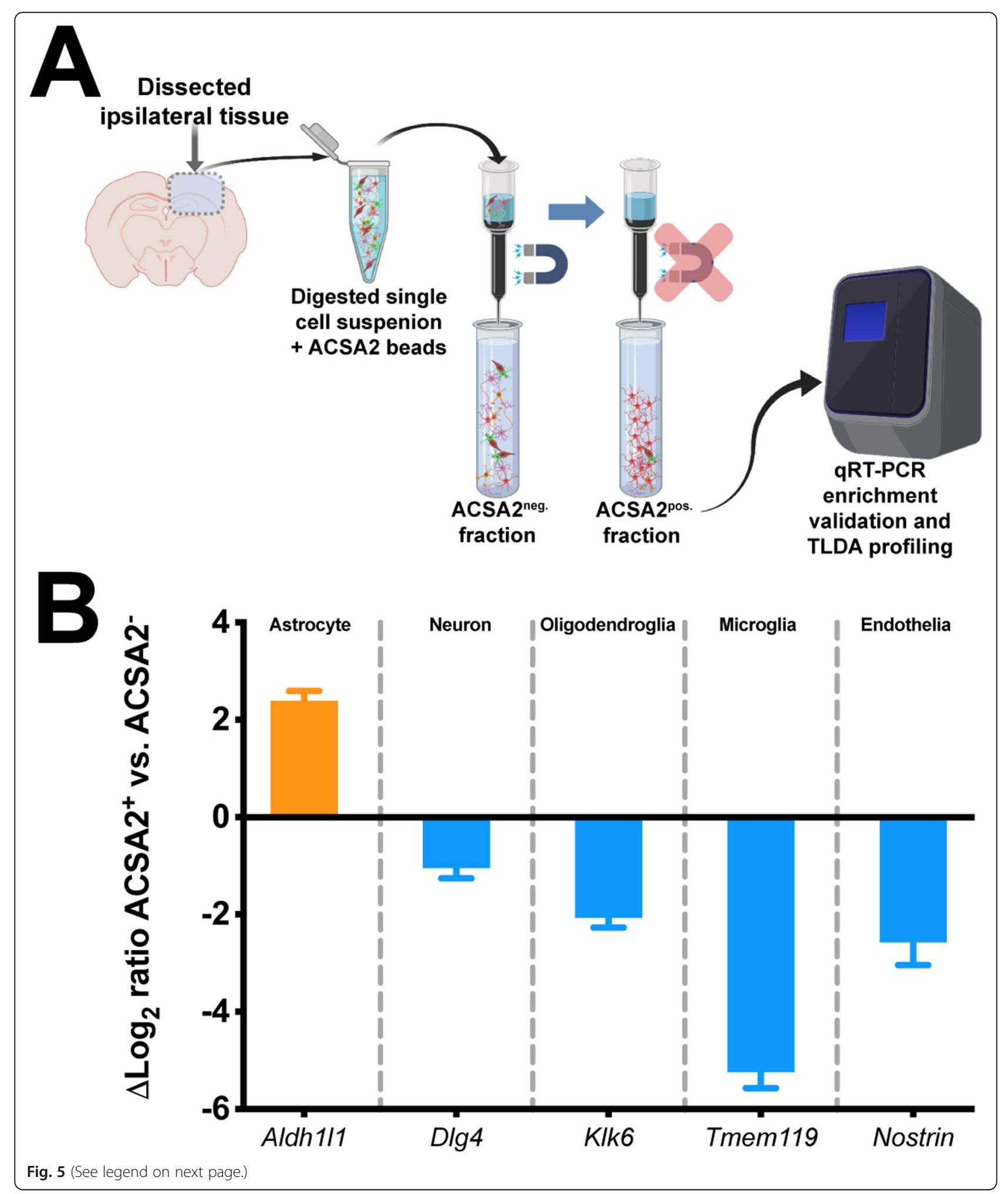


(See figure on previous page.)

Fig. 5 Validation of ACSA-2 astrocyte cell enrichment from brain tissue. a Generalized workflow for ACSA-2 magnetic bead enrichment of astrocytes from the injured brain parenchyma. Digested cell suspensions were labeled with the ACSA-2 magnetic bead before being placed in the magnetic column for removal of non-specific cells, which were collected into a tube as the ACSA-2 ${ }^{\text {neg. }}$ fraction. Removal of the column from the magnetic stand allowed the flow-through of the retained ACSA-2 astrocytes to be collected as the ACSA-2 ${ }^{\text {pos. }}$ fraction. RNA from both fractions was harvested to examine gene expression endpoints. $\mathbf{b}$ Gene expression analyses using putative markers of five neural tissue subsets: astrocyte (Aldh1/1), neuronal (D/g4), oligodendroglial (Klk6), microglial (Tmem119), and endothelial (Nostrin). These data demonstrate a significant induction of putative astrocyte signature (orange bar), with little signature of other cell populations (blue bars). $\log _{2}$ fold change is a ratio of ACSA-2 ${ }^{\text {pos }}$ to ACSA-2 ${ }^{\text {neg }}$. TLDA, Taqman low density array cards

some regional specificity in terms of age-exacerbated reactive astrogliosis, such that the hippocampus appeared to be more vulnerable than the neocortex at several time points in the aged brain, compared to young mice.

Our present findings demonstrating an age-related increase in clasmatodendrosis are corroborated by previous work examining human brains [45]. We observed the aggregation of this morphologically distinct subset of astrocytes confined to the stratum radiatum of the CA1 $\beta$ region of the hippocampus, which recapitulates previous work in rodents demonstrating this as a susceptible region for accumulating these astrogliopathies [62-64]. Although the exact role or cause of this pathology is not well-defined, recent reports have demonstrated corollaries with






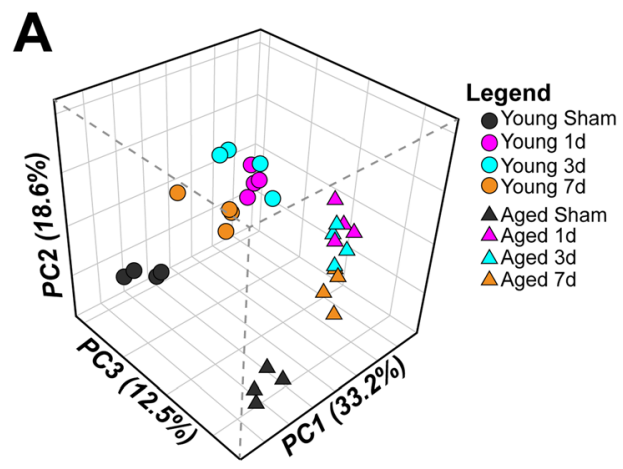

B
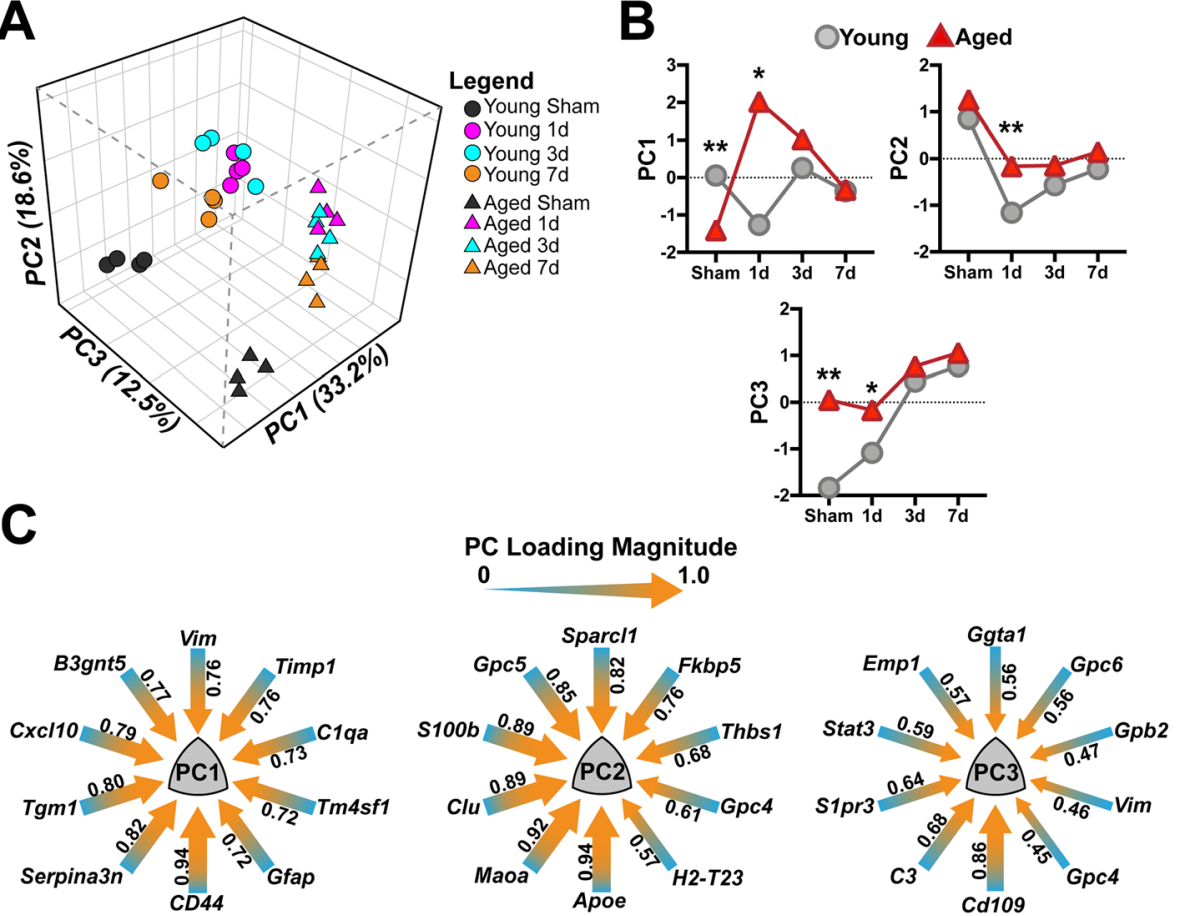

D
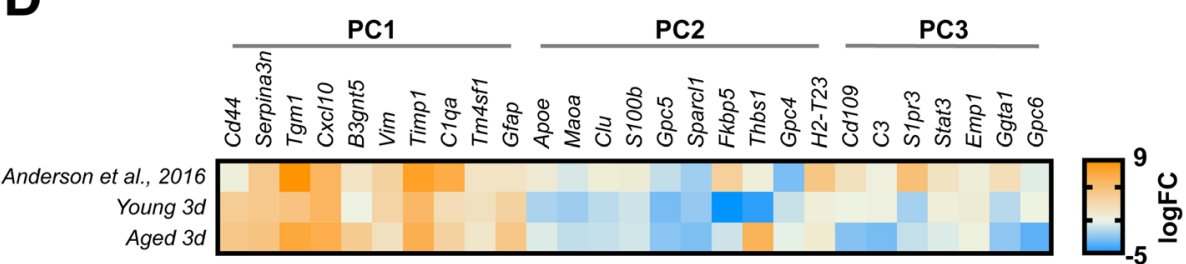

Fig. 7 Astrocyte-specific response patterns to age and TBI revealed by principal components analysis (PCA). a Multivariate dimensional reduction by PCA uncovered 3 orthogonal PC groups that cumulatively accounted for $64.3 \%$ of the total variance. Within this multidimensional space, PC1 (33.2\% variance) reflected a primarily TBI-induced response, while PC2 (18.6\% variance) reflected some of the temporal responses within the dataset, and lastly PC3 (12.5\% variance) reflected an age-related effect. $\mathbf{b}$ Temporal trajectories of the mean PC score for each group in each of the three PCs identified. Data were analyzed using two-way ANOVA with Sidak-Holm correction for examining pairwise interactions for each time interval. Data points represent the mean PC score for each interval and age. Young, gray circles; Aged, red triangles. c PC loading magnitudes for the top 10 genes $>|.45|$ loading for each of the three PCs. Arrow gauge (thickness) is proportional to loading magnitude, with heat (color) representing loading directionality toward 1.0. d Comparison of the $\log _{2}$ fold change (logFC) from "SCI WT Astrocyte" vs. "Uninjured WT Astrocytes" from Anderson et al. versus mean logFC of young 3-day and aged 3-day cohorts from the current study. Comparing 3-day TBI responses from both young and aged cohorts with SCl-induced responses reveals a striking similarity in PC1-related profiles between the two datasets. Data were arranged within the previously defined PCs from above; duplicates of genes from PCs (e.g., PC1 Vim) were removed for clarity. Additionally, no data were found in the Anderson et al. dataset for Gpb2, which was also removed for clarity

senescence, autophagy, metabolic dysfunction, ER stress, and NFkB signaling $[62,63,65,66]$. Moreover, our findings potentially highlight a trauma-induced turnover response occurring at the 3-day post-injury interval; however, our current analyses are unable to distinguish if this represents a loss in the number of these cells or that a portion of the population transiently loses this morphology.

Aqp4 expression and distribution across the perivascular environment has been previously shown to be significantly disrupted as a function of aging alone in a rodent model of aging and in human post-mortem examinations $[67,68]$. Critically, Aqp4 serves as an integral water channel in the brain's glymphatic system for waste material clearance by facilitating the exchange between both CSF and interstitial fluids [69]. These dynamics are largely associated with the concentrated Aqp4 localization along the perivascular and subpial astrocyte endfoot membranes [70]. Moreover, the transient responses we observed in response to TBI may point toward a refractory response wherein astrocytes have lost their vascular-related homeostatic capacity in the aged brain, potentially implicating transient impairment 
of solute clearance mechanisms, vascular tone, and cerebral blood flow.

Recent work profiling the transcriptome of aged astrocytes has demonstrated altered expression in pathways associated with synaptic function, inflammatory disposition, and disproportionate responses to LPS-mediated insult $[28,29]$. In essence, this previous work implicates aged astrocytes as a "primed" cell population, similar to how microglia have been shown to become predisposed to insult or stress in the aged brain [71]. At least in the context of our narrow geneset, our current findings would be in agreement with the potential for TBI to initiate a phenotype outside these bounds, or as a continuum of these stimulus-specific bins [36]. Several of these unique responses included age-related shifts in expression for inflammatory markers CXCL10, CD44, and C1qa. Our data demonstrate a similar exacerbated CXCL10 response in aged astrocytes due to TBI. CXCL10 has been shown to modulate chemotaxis, initiate cross-talk between immunoreactive microglia and astrocytes [72], and was previously documented to have an exacerbated response in aged astrocytes following LPSinduced inflammatory challenge [28]. CD44 defines astrocyte precursors $[73,74]$, their tissue heterogeneity in the adult brain [75], and may identify astrocytes that are both injury and disease responsive [76-78]. Interestingly, recent work suggests that the acquisition of CD44 on astrocytes is indicative of a regressive phenotype toward an immature state [76]. Collectively, our findings of increased inflammatory response paired with reductions in synaptic support markers, such as Gpc6 and the upregulation of Clqa, a complement protein that has been shown to play a role in tagging synapses for their selective removal [11], may suggest a maladaptive environment where neurons and/or their synapses are highly susceptible to detrimental phenotypes by aged astrocytes; however, mechanistic studies are needed to validate these findings.

Our multivariate approach to interpreting these responses yielded several uniquely correlating gene expression phenotypes, which show that in both the uninjured and 1-day post-injury timepoints that aged mice had significantly altered outcomes within these multivariate domains, compared to young. Comparatively, when we examined our injury-induced responses that displayed some exacerbation in the context of age (e.g., PC1) with transcriptome profiling of injured astrocytes from the spinal cord, there was an analogous resemblance to these data [20]. Therefore, concordance between these findings may point toward a conserved reactivity among astrocytes following contusion, irrespective of CNS locale.

Despite concordance between our findings and several previous reports for aging and injury, there remain several caveats with our design and resultant dataset that warrant acknowledgement as well as open exciting future directions to explore. First, our antibody-based enrichment method may induce bias in the types of astrocytes, only ACSA-2 ${ }^{\text {pos }}$ enriched from our samples, compared to recent methods using a global astrocytespecific (e.g., Aldh1l1) promoter to drive mechanisms for polyribosomal RNA purification (e.g., bacTRAP or RiboTag), as was previously described for profiling agerelated responses for astrocytes [28, 29]. Similarly, there is known heterogeneity in astrocyte populations in homeostasis [23] as well as a function of distance from injury (for review see [26]). Therefore, it is possible that even though we observed significant differences in our aged cohort across several timepoints, there may be even more nuanced, but disease-relevant responses that would be detected using either of the ribosomal affinity purification techniques or single cell analyses in tandem with whole transcriptome profiling. Secondly, our approach used mixed gender groups. Recent work in young adult mice using the CCI method that utilized higher velocity and tissue penetrance parameters, compared to our current design, demonstrated that male mice had significantly exaggerated increases in GFAP reactive gliosis, compared to females at 1,3 , and 7 days post-injury [79]. Although our design was not powered to detect sex as an interaction in our planned contrasts, and distribution of quantified values for each endpoint did not reveal any visual trends for separation by sex, this does not preclude the potential for an a priori powered design from revealing sex-based effects. Therefore, future work to determine if our CCI model has sex-related effects in aged mice is warranted. Lastly, our focus in this study was to understand the acute responses of astrocytes, as a complement to our previous work showing altered neuroinflammatory responses due to injury and/or advanced age chronically. Future critical work examining the persistence of chronic dysfunctional responses in aged astrocytes may shed light on how these cells are affecting unique signaling domains underlying chronic neuronal, synaptic, and cognitive impairments seen in our TBI model [31,33].

\section{Conclusion}

Our results demonstrate that advanced age predisposes astrocytes to acquire degenerative physiologic properties, with progressively aberrant morphological responses to TBI, compared to young. Further, we demonstrate that in the aged brain, the hippocampal formation appears to be more vulnerable to TBI-induced morphological changes in astrocyte reactivity, compared to the neocortex. In terms of the heterogeneous molecular responses attributed to astrocytes, our findings parallel previous reports in terms of their age-related altered basal signature, which is exacerbated by injury. Interestingly, TBIinduced molecular signatures of astrocytes show a surprising conservation of phenotypes when compared with 
astrocytes profiled after SCI. Taken together, our initial characterization of aged astrocytes' response to TBI begs the question as to whether these disproportionate motifs are responsible for exaggerated functional outcomes in the aged brain, and importantly, whether mechanistically targeting astrocytes specifically to alter some of these responses would offer functional recovery.

\section{Abbreviations}

AD: Alzheimer's disease; Aqp4: Aquaporin4; ACSA-2: Astrocyte cell surface antigen 2; BBB: Blood brain barrier; CBF: Cerebral blood flow; CSF: Cerebral spinal fluid; CTE: Chronic traumatic encephalopathy; CCI: Controlled cortical impact; CA1: Cornu Ammonis area 1; DAMPs: Damage-associated molecular patterns; GFAP: Glial fibrillary acid protein; HPC: Hippocampus; LPS: Lipopolysaccharide; MCAO: Middle cerebral artery occlusion; NGS: Normal goat serum; PFA: Paraformaldehyde; PBS: Phosphate buffered saline; PCA: Principal component analysis; SCl: Spinal cord injury; TLDA: Taqman low density array; TBI: Traumatic brain injury

\section{Acknowledgements}

We also wish to thank Edgardo Dimayuga for the excellent technical assistance toward the completion of this project.

\section{Authors' contributions}

ANE and JMM designed the research studies. ANE, AAG, ADB, and JMM performed the experiments. LJVE contributed the critical reagents and provided the manuscript edits. ANE, ADB, and JMM analyzed the data. ANE and JMM wrote the manuscript. All authors have read and approved the final manuscript.

\section{Funding}

This project was supported by the National Center for Research Resources and the National Center for Advancing Translational Sciences and National Institute on Aging, National Institutes of Health, through Grants R01NS103785 (ADB), R00AG044445 (ADB), UL1TR001998 pilot award (JMM), and R21AG058006 (JMM). The content is solely the responsibility of the authors and does not necessarily represent the official views of the National Institutes of Health.

\section{Availability of data and materials}

The datasets generated and/or analyzed during this study are available from the corresponding author on reasonable request.

\section{Ethics approval and consent to participate}

All procedures involving animals for this study were approved by the Institutional Animal Care and Use Committee of the University of Kentucky.

\section{Consent for publication}

Not applicable.

\section{Competing interests}

The authors declare that they have no competing interests.

\section{Author details}

${ }^{1}$ Sanders-Brown Center on Aging, University of Kentucky, Room 433 , Sanders-Brown Bldg., 800 S. Limestone Street, Lexington, KY 40536, USA. 2Department of Neuroscience, University of Kentucky, Lexington, KY 40536, USA. ${ }^{3}$ Spinal Cord and Brain Injury Research Center, University of Kentucky, Lexington, KY 40536, USA.

Received: 14 November 2019 Accepted: 1 April 2020

\section{Published online: 14 April 2020}

\section{References}

1. Fleminger S, Oliver DL, Lovestone S, Rabe-Hesketh S, Giora A. Head injury as a risk factor for Alzheimer's disease: the evidence 10 years on; a partial replication. J Neurol Neurosurg Psychiatry. 2003;74:857-62.

2. Jellinger KA. Traumatic brain injury as a risk factor for Alzheimer's disease. Neurol Neurosurg Psychiatry. 2004;75:511-2.
3. Schönberger M, Ponsford J, Reutens $D$, Beare $R, O$ apos Sullivan R: The relationship between age, injury severity, and MRI findings after traumatic brain injury. J Neurotrauma. 2009;26:2157-2167.

4. Graves AB, White E, Koepsell TD, Reifler BV, van Belle G, Larson EB, Raskind $M$. The association between head trauma and Alzheimer's disease. American journal of epidemiology. 1990;131:491-501.

5. Gavett BE, Stern RA, Cantu RC, Nowinski CJ, McKee AC. Mild traumatic brain injury: a risk factor for neurodegeneration. Alzheimers Res Ther. 2010;2:18.

6. Uryu K, Chen X-H, Martinez D, Browne KD, Johnson VE, Graham DI, Lee VMY, Trojanowski JQ, Smith DH. Multiple proteins implicated in neurodegenerative diseases accumulate in axons after brain trauma in humans. Experimental neurology. 2007;208:185-92.

7. Johnson VE, Stewart W, Smith DH. Widespread tau and amyloid-beta pathology many years after a single traumatic brain injury in humans. LongTerm AD-Like Pathology after Single TBI. 2011;22:142-9.

8. Coulter DA, Eid T. Astrocytic regulation of glutamate homeostasis in epilepsy. Glia. 2012;60:1215-26.

9. Lalo U, Palygin O, Rasooli-Nejad S, Andrew J, Haydon PG, Pankratov Y. Exocytosis of ATP from astrocytes modulates phasic and tonic inhibition in the neocortex. PLoS Biol. 2014;12:e1001747.

10. Frieler RA, Nadimpalli S, Boland LK, Xie A, Kooistra LJ, Song J, Chung Y, Cho KW, Lumeng CN, Wang MM, Mortensen RM. Depletion of macrophages in CD11b diphtheria toxin receptor mice induces brain inflammation and enhances inflammatory signaling during traumatic brain injury. Brain Res. 1624;2015:103-12.

11. Allen NJ, Eroglu C. Cell biology of astrocyte-synapse interactions. Neuron. 2017:96:697-708.

12. Takano T, Tian GF, Peng W, Lou N, Libionka W, Han X, Nedergaard M. Astrocyte-mediated control of cerebral blood flow. Nat Neurosci. 2006;9:260-7.

13. Seifert G, Steinhauser C. Neuron-astrocyte signaling and epilepsy. Exp Neurol. 2013;244:4-10

14. Clarke LE, Barres BA. Emerging roles of astrocytes in neural circuit development. Nat Rev Neurosci. 2013;14:311-21.

15. Stevens B, Allen NJ, Vazquez LE, Howell GR, Christopherson KS, Nouri N, Micheva KD, Mehalow AK, Huberman AD, Stafford B, et al. The classical complement cascade mediates CNS synapse elimination. Cell. 2007:131:1164-78.

16. Sofroniew MV. Astrocyte barriers to neurotoxic inflammation. Nat Rev Neurosci. 2015:16:249-63.

17. Cahoy JD, Emery B, Kaushal A, Foo LC, Zamanian JL, Christopherson KS, Xing Y, Lubischer JL, Krieg PA, Krupenko SA, et al. A transcriptome database for astrocytes, neurons, and oligodendrocytes: a new resource for understanding brain development and function. J Neurosci. 2008;28:264-78.

18. Sofroniew MV, Vinters HV. Astrocytes: biology and pathology. Acta Neuropathologica. 2009;119:7-35

19. Myer DJ, Gurkoff GG, Lee SM, Hovda DA, Sofroniew MV. Essential protective roles of reactive astrocytes in traumatic brain injury. Brain. 2006;129:2761-72

20. Anderson MA, Burda JE, Ren Y, Ao Y, O'Shea TM, Kawaguchi R, Coppola G, Khakh BS, Deming TJ, Sofroniew MV. Astrocyte scar formation aids central nervous system axon regeneration. Nature. 2016:532:195-200.

21. Burda JE, Sofroniew MV. Reactive gliosis and the multicellular response to CNS damage and disease. Neuron. 2014;81:229-48.

22. Zamanian JL, Xu L, Foo LC, Nouri N, Zhou L, Giffard RG, Barres BA. Genomic analysis of reactive astrogliosis. The Journal of neuroscience : the official journal of the Society for Neuroscience. 2012;32:6391-410.

23. Lin C-CJ YK, Hatcher A, Huang T-W, Lee HK, Carlson J, Weston MC, Chen F, Zhang $Y$, Zhu W, et al. Identification of diverse astrocyte populations and their malignant analogs. Nat Neurosci. 2017;20:396-405.

24. Shandra O, Winemiller AR, Heithoff BP, Munoz-Ballester C, George KK, Benko MJ, Zuidhoek IA, Besser MN, Curley DE, Edwards GF, et al. Repetitive diffuse mild traumatic brain injury causes an atypical astrocyte response and spontaneous recurrent seizures. J Neurosci. 2019;39:1944-63.

25. Wanner IB, Anderson MA, Song B, Levine J, Fernandez A, Gray-Thompson Z, Ao $Y$, Sofroniew MV. Glial scar borders are formed by newly proliferated, elongated astrocytes that interact to corral inflammatory and fibrotic cells via STAT3-dependent mechanisms after spinal cord injury. J Neurosci. 2013; 33:12870-86.

26. Burda JE, Bernstein AM, Sofroniew MV. Astrocyte roles in traumatic brain injury. Exp Neurol. 2016;275(Pt 3):305-15.

27. Salminen A, Ojala J, Kaarniranta K, Haapasalo A, Hiltunen M, Soininen H. Astrocytes in the aging brain express characteristics of senescenceassociated secretory phenotype. Eur J Neurosci. 2011;34:3-11. 
28. Clarke LE, Liddelow SA, Chakraborty C, Munch AE, Heiman M, Barres BA. Normal aging induces A1-like astrocyte reactivity. Proc Natl Acad Sci U S A. 2018;115:E1896-905

29. Boisvert MM, Erikson GA, Shokhirev MN, Allen NJ. The aging astrocyte transcriptome from multiple regions of the mouse brain. Cell Rep. 2018;22:269-85.

30. Morganti JM, Riparip LK, Chou A, Liu S, Gupta N, Rosi S. Age exacerbates the CCR2/5-mediated neuroinflammatory response to traumatic brain injury. J Neuroinflammation. 2016;13:80.

31. Chou A, Krukowski K, Morganti JM, Riparip LK, Rosi S. Persistent infiltration and impaired response of peripherally-derived monocytes after traumatic brain injury in the aged brain. Int J Mol Sci. 2018;19(6):E1616.

32. Kumar A, Stoica BA, Sabirzhanov B, Burns MP, Faden Al, Loane DJ. Traumatic brain injury in aged animals increases lesion size and chronically alters microglial/macrophage classical and alternative activation states. Neurobiol Aging. 2013;34:1397-411.

33. Morganti JM, Jopson TD, Liu S, Riparip LK, Guandique CK, Gupta N, Ferguson AR, Rosi S. CCR2 antagonism alters brain macrophage polarization and ameliorates cognitive dysfunction induced by traumatic brain injury. J Neurosci. 2015;35:748-60.

34. Morganti JM, Riparip LK, Rosi S. Call off the dog(ma): M1/M2 polarization is concurrent following traumatic brain injury. PLoS One. 2016;11:e0148001.

35. Siebold L, Obenaus A, Goyal R. Criteria to define mild, moderate, and severe traumatic brain injury in the mouse controlled cortical impact model. Exp Neurol. 2018;310:48-57.

36. Liddelow SA, Guttenplan KA, Clarke LE, Bennett FC, Bohlen CJ, Schirmer $L$, Bennett ML, Münch AE, Chung W-S, Peterson TC, et al. Neurotoxic reactive astrocytes are induced by activated microglia. Nature. 2017;541:481-7.

37. Pekny M. Astrocytic intermediate filaments: lessons from GFAP and vimentin knock-out mice. Prog Brain Res. 2001;132:23-30.

38. Pekny M, Lane EB. Intermediate filaments and stress. Exp Cell Res. 2007:313:2244-54.

39. Pekny M, Pekna M. Astrocyte reactivity and reactive astrogliosis: costs and benefits. Physiological reviews. 2014;94:1077-98.

40. Vives $V$, Alonso $G$, Solal $A C$, Joubert $D$, Legraverend C. Visualization of S100B-positive neurons and glia in the central nervous system of EGFP transgenic mice. The Journal of comparative neurology. 2003;457:404-19.

41. Penfield W. Neuroglia and microglia-the interstitial tissue of the central nervous system. New York: Hoeber; 1928.

42. Akiguchi I, Tomimoto $H$, Suenaga $T$, Wakita H, Budka H. Alterations in glia and axons in the brains of Binswanger's disease patients. Stroke. 1997;28: 1423-9.

43. Tomimoto $\mathrm{H}$, Akiguchi I, Wakita H, Suenaga T, Nakamura S, Kimura J. Regressive changes of astroglia in white matter lesions in cerebrovascular disease and Alzheimer's disease patients. Acta Neuropathol. 1997:94:146-52.

44. Hulse RE, Winterfield J, Kunkler PE, Kraig RP. Astrocytic clasmatodendrosis in hippocampal organ culture. Glia. 2001;33:169-79.

45. Chen A, Akinyemi RO, Hase Y, Firbank MJ, Ndung'u MN, Foster V, Craggs LL, Washida K, Okamoto Y, Thomas AJ, et al. Frontal white matter hyperintensities, clasmatodendrosis and gliovascular abnormalities in ageing and post-stroke dementia. Brain. 2016;139:242-58.

46. Sahlas DJ, Bilbao JM, Swartz RH, Black SE. Clasmatodendrosis correlating with periventricular hyperintensity in mixed dementia. Ann Neurol. 2002;52:378-81

47. Wilcock DM, Vitek MP, Colton CA. Vascular amyloid alters astrocytic water and potassium channels in mouse models and humans with Alzheimer's disease. Neuroscience. 2009;159:1055-69.

48. Hsu ET, Gangolli M, Su S, Holleran L, Stein TD, Alvarez VE, McKee AC, Schmidt RE, Brody DL. Astrocytic degeneration in chronic traumatic encephalopathy. Acta Neuropathol. 2018;136:955-72.

49. Fukuda AM, Badaut J. Aquaporin 4: a player in cerebral edema and neuroinflammation. J Neuroinflammation. 2012:9:279.

50. ielsen S, Nagelhus EA, Amiry-Moghaddam M, Bourque C, Agre P, Ottersen OP. Specialized membrane domains for water transport in glial cells: highresolution immunogold cytochemistry of aquaporin-4 in rat brain. $J$ Neurosci. 1997;17:171-80

51. Liddelow SA, Barres BA. Reactive astrocytes: production, function, and therapeutic potential. Immunity. 2017:46:957-67.

52. Kantzer CG, Boutin C, Herzig ID, Wittwer C, Reiß S, Tiveron MC, Drewes J, Rockel TD, Ohlig S, Ninkovic J, et al. Anti-ACSA-2 defines a novel monoclonal antibody for prospective isolation of living neonatal and adult astrocytes. Glia. 2017;65:990-1004.
53. Feldmann M, Pathipati $P$, Sheldon RA, Jiang $X$, Ferriero DM. Isolating astrocytes and neurons sequentially from postnatal murine brains with a magnetic cell separation technique. J Biological Methods. 2014;1:e11.

54. Batiuk MY, de Vin F, Duque SI, Li C, Saito T, Saido T, Fiers M, Belgard TG, Holt MG. An immunoaffinity-based method for isolating ultrapure adult astrocytes based on ATP1B2 targeting by the ACSA-2 antibody. J Biol Chem. 2017;292:8874-91.

55. Sandhir R, Onyszchuk G, Berman NEJ. Exacerbated glial response in the aged mouse hippocampus following controlled cortical impact injury. Experimental neurology. 2008;213:372-80.

56. van Eldik LJ, Wainwright MS. The Janus face of glial-derived S100B: beneficial and detrimental functions in the brain. Restorative neurology and neuroscience. 2003:21:97-108.

57. Sorci G, Riuzzi F, Arcuri C, Bianchi R, Brozzi F, Tubaro C, Giambanco I, Donato R. The many faces of S100B protein: when an extracellular factor inactivates its own receptor and activates another one. Ital J Anat Embryol. 2010;115:147-51.

58. Ahlemeyer B, Beier H, Semkova I, Schaper C, Krieglstein J. S-100beta protects cultured neurons against glutamate- and staurosporine-induced damage and is involved in the antiapoptotic action of the $5 \mathrm{HT}(1 \mathrm{~A})$-receptor agonist, Bay x 3702. Brain research. 2000;858:121-8.

59. Selinfreund RH, Barger SW, Pledger WJ, Van Eldik LJ. Neurotrophic protein S100 beta stimulates glial cell proliferation. Proc Natl Acad Sci U S A. 1991; 88:3554-8.

60. Kleindienst A, Harvey HB, Rice AC, Muller C, Hamm RJ, Gaab MR, Bullock MR. Intraventricular infusion of the neurotrophic protein S100B improves cognitive recovery after fluid percussion injury in the rat. J Neurotrauma. 2004:21:541-7.

61. Kleindienst A, McGinn MJ, Harvey HB, Colello RJ, Hamm RJ, Bullock MR. Enhanced hippocampal neurogenesis by intraventricular S100B infusion is associated with improved cognitive recovery after traumatic brain injury. Journal of neurotrauma. 2005;22:645-55.

62. Ryu HJ, Kim J-E, Yeo S-I, Kang T-C. p65/RelA-Ser529 NF-kB subunit phosphorylation induces autophagic astroglial death (Clasmatodendrosis) following status epilepticus. Cellular and Molecular Neurobiology. 2011;31:1071-8.

63. Sakai K, Fukuda T, Iwadate K. Beading of the astrocytic processes (clasmatodendrosis) following head trauma is associated with protein degradation pathways. Brain injury: [BI]. 2013;27:1692-7.

64. Kim DS, Kim JE, Kwak SE, Choi KC, Kim DW, Kwon OS, Choi SY, Kang TC. Spatiotemporal characteristics of astroglial death in the rat hippocampoentorhinal complex following pilocarpine-induced status epilepticus. J Comp Neurol. 2008;511:581-98.

65. Cerbai F, Lana D, Nosi D, Petkova-Kirova P, Zecchi S, Brothers HM, Wenk GL, Giovannini MG. The neuron-astrocyte-microglia triad in normal brain ageing and in a model of neuroinflammation in the rat hippocampus. PloS One. 2012; 7:e45250.

66. Kim J-H, Ko P-W, Lee H-W, Jeong J-Y, Lee M-G, Kim J-H, Lee W-H, Yu R, Oh W-J, Suk K. Astrocyte-derived lipocalin-2 mediates hippocampal damage and cognitive deficits in experimental models of vascular dementia. Glia. 2017;65:1471-90.

67. Kress BT, Iliff JJ, Xia M, Wang M, Wei HS, Zeppenfeld D, Xie L, Kang H, Xu Q Liew JA, et al. Impairment of paravascular clearance pathways in the aging brain. Annals of neurology. 2014:76:845-61.

68. Zeppenfeld DM, Simon M, Haswell JD, D'Abreo D, Murchison C, Quinn JF, Grafe MR, Woltjer RL, Kaye J, lliff JJ. Association of perivascular localization of aquaporin-4 with cognition and Alzheimer disease in aging brains. JAMA Neurol. 2017;74:91-9.

69. Iliff JJ, Wang M, Liao Y, Plogg BA, Peng W, Gundersen GA, Benveniste H, Vates GE, Deane R, Goldman SA, et al. A paravascular pathway facilitates CSF flow through the brain parenchyma and the clearance of interstitial solutes, including amyloid. Sci Transl Med. 2012;4:147ra111-147ra111.

70. Erlend A, Nagelhus OPO. Physiological roles of aquaporin-4 in brain. Physiological Rev. 2013;93:1543-62.

71. Norden DM, Godbout JP. Review: microglia of the aged brain: primed to be activated and resistant to regulation. Neuropathol Appl Neurobiol. 2013;39:19-34.

72. Domingues HS, Portugal CC, Socodato R, Relvas JB. Oligodendrocyte, astrocyte, and microglia crosstalk in myelin development, damage, and repair. Front Cell Dev Biol. 2016:4:71.

73. Liu Y, Han SSW, Wu Y, Tuohy TMF, Xue H, Cai J, Back SA, Sherman LS, Fischer I, Rao MS. CD44 expression identifies astrocyte-restricted precursor cells. Developmental Biology. 2004;276:31-46. 
74. Naruse M, Shibasaki K, Yokoyama S, Kurachi M, Ishizaki Y. Dynamic changes of CD44 expression from progenitors to subpopulations of astrocytes and neurons in developing cerebellum. PloS One. 2013;8:e53109.

75. Sosunov AA, Wu X, Tsankova NM, Guilfoyle E, McKhann GM, Goldman JE. Phenotypic heterogeneity and plasticity of isocortical and hippocampal astrocytes in the human brain. J Neurosci. 2014;34:2285-98.

76. Gabel S, Koncina E, Dorban G, Heurtaux T, Birck C, Glaab E, Michelucci A, Heuschling P, Grandbarbe L. Inflammation promotes a conversion of astrocytes into neural progenitor cells via NF-kappaB activation. Mol Neurobiol. 2016;53:5041-55.

77. Matsumoto T, Imagama S, Hirano K, Ohgomori T, Natori T, Kobayashi K, Muramoto A, Ishiguro N, Kadomatsu K. CD44 expression in astrocytes and microglia is associated with ALS progression in a mouse model. Neuroscience letters. 2012;520:115-20.

78. Sosunov AA, Guilfoyle E, Wu X, McKhann GM, Goldman JE. Phenotypic conversions of "protoplasmic" to "reactive" astrocytes in Alexander disease. J Neuroscience. 2013;33:7439-50.

79. Villapol S, Loane DJ, Burns MP. Sexual dimorphism in the inflammatory response to traumatic brain injury. Glia. 2017;63:1966-16.

\section{Publisher's Note}

Springer Nature remains neutral with regard to jurisdictional claims in published maps and institutional affiliations.

Ready to submit your research? Choose BMC and benefit from:

- fast, convenient online submission

- thorough peer review by experienced researchers in your field

- rapid publication on acceptance

- support for research data, including large and complex data types

- gold Open Access which fosters wider collaboration and increased citations

- maximum visibility for your research: over $100 \mathrm{M}$ website views per year

At $\mathrm{BMC}$, research is always in progress.

Learn more biomedcentral.com/submissions 